\title{
Observational Evidence for Extended Emission to GW170817
}

\author{
Maurice H.P.M. van Putten \\ Physics and Astronomy, Sejong University \\ Seoul, South Korea
}

Aspen 2019 GWPop 2019, Feb 9-15

Feb 132019 


\section{A multi-messenger window to the Universe}

Fermi

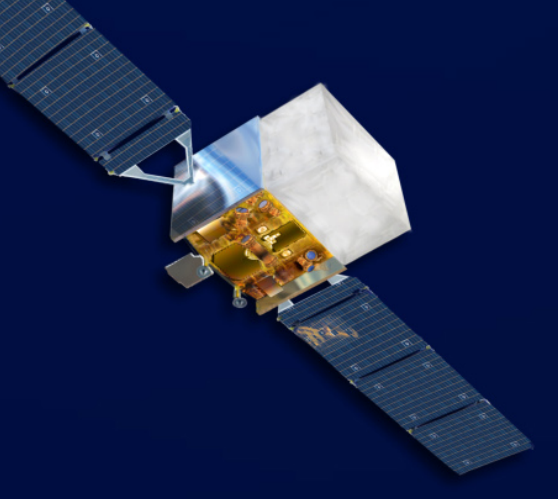

\section{LIGO}

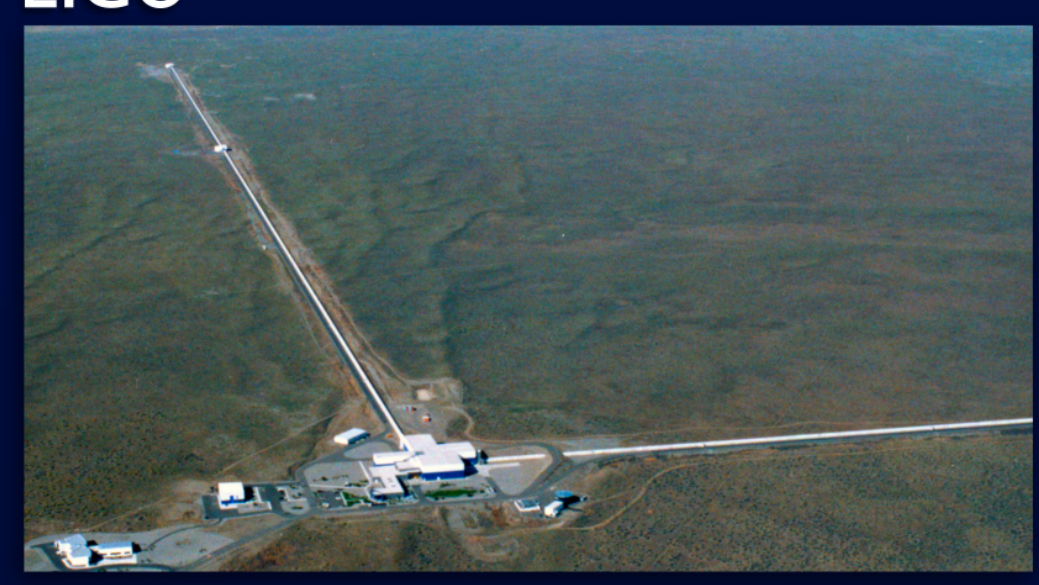

Gamma rays, 50 to $300 \mathrm{keV}$
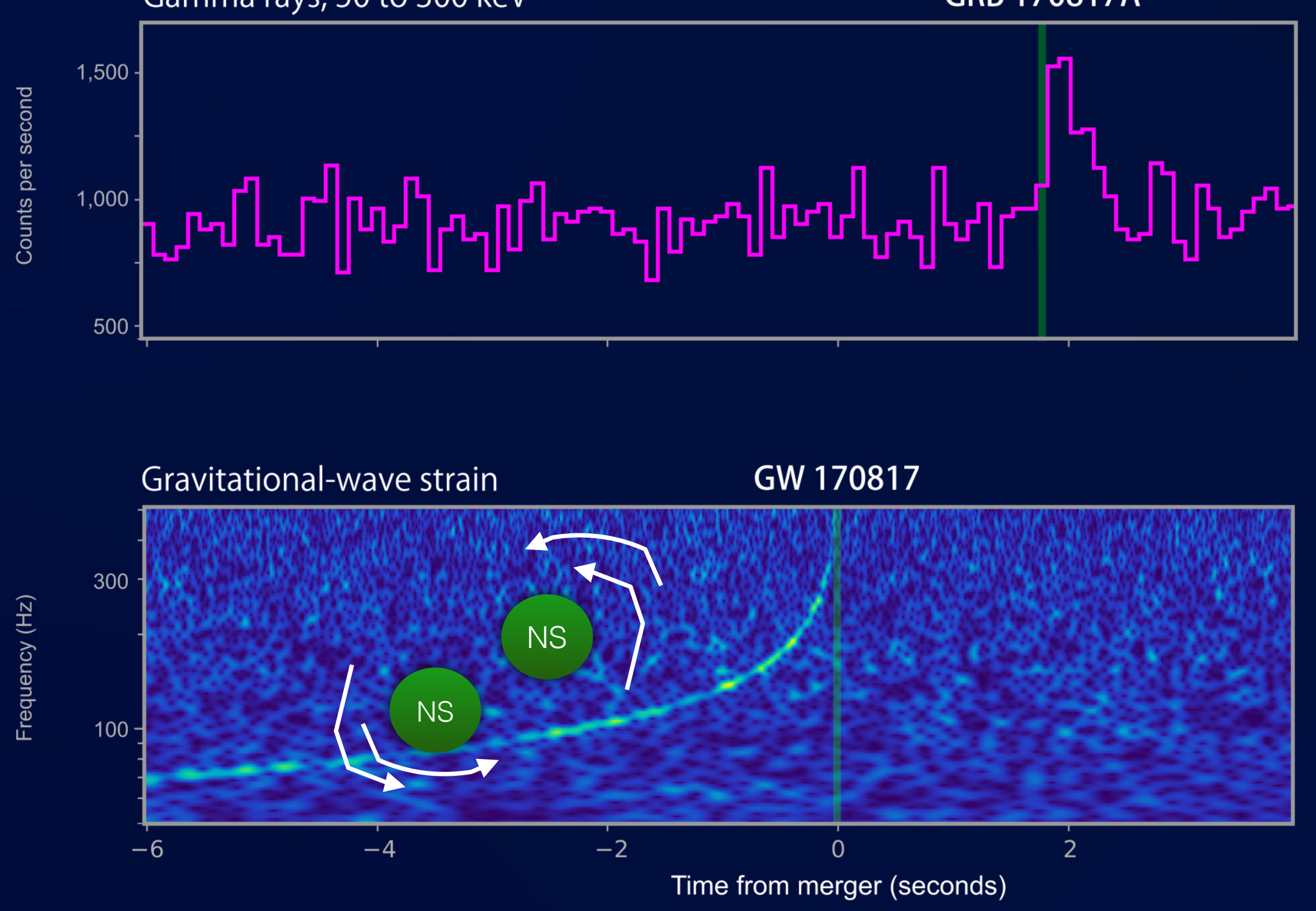


\section{GW170817: what happened?}

Fermi

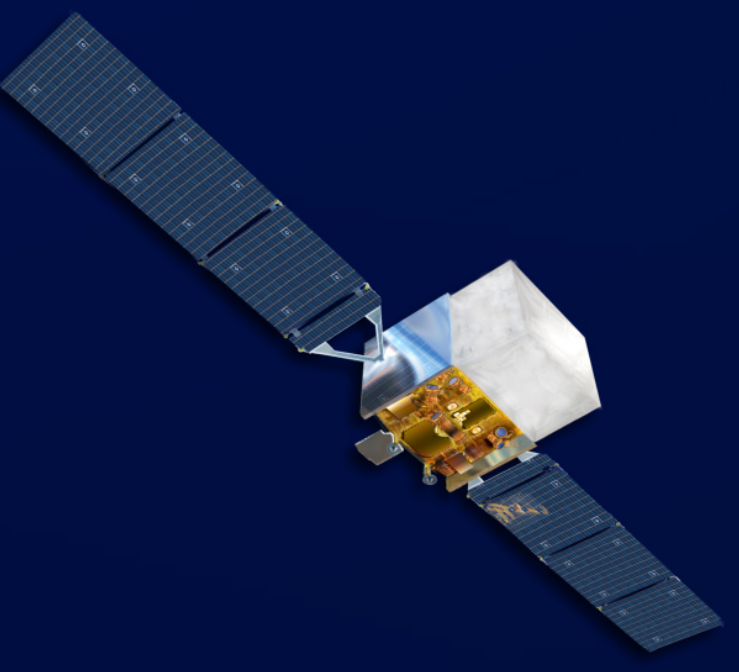

\section{LIGO}

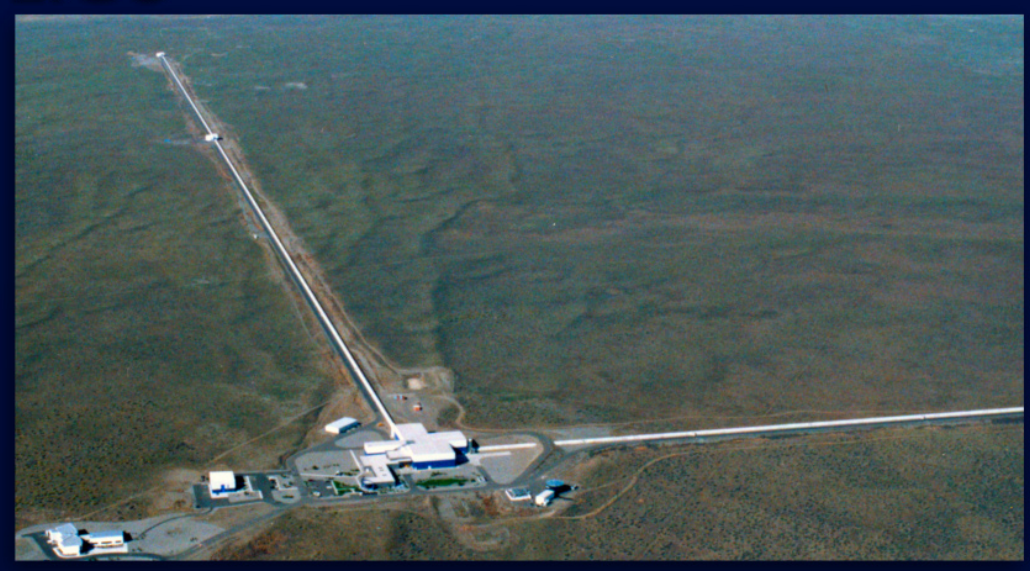

Gamma rays, 50 to $300 \mathrm{keV}$

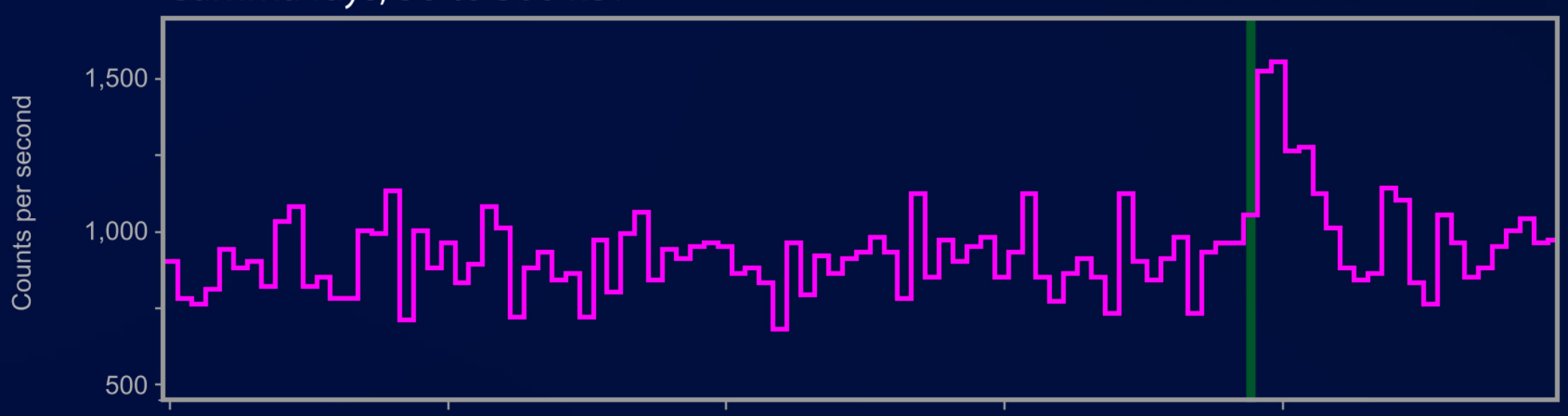

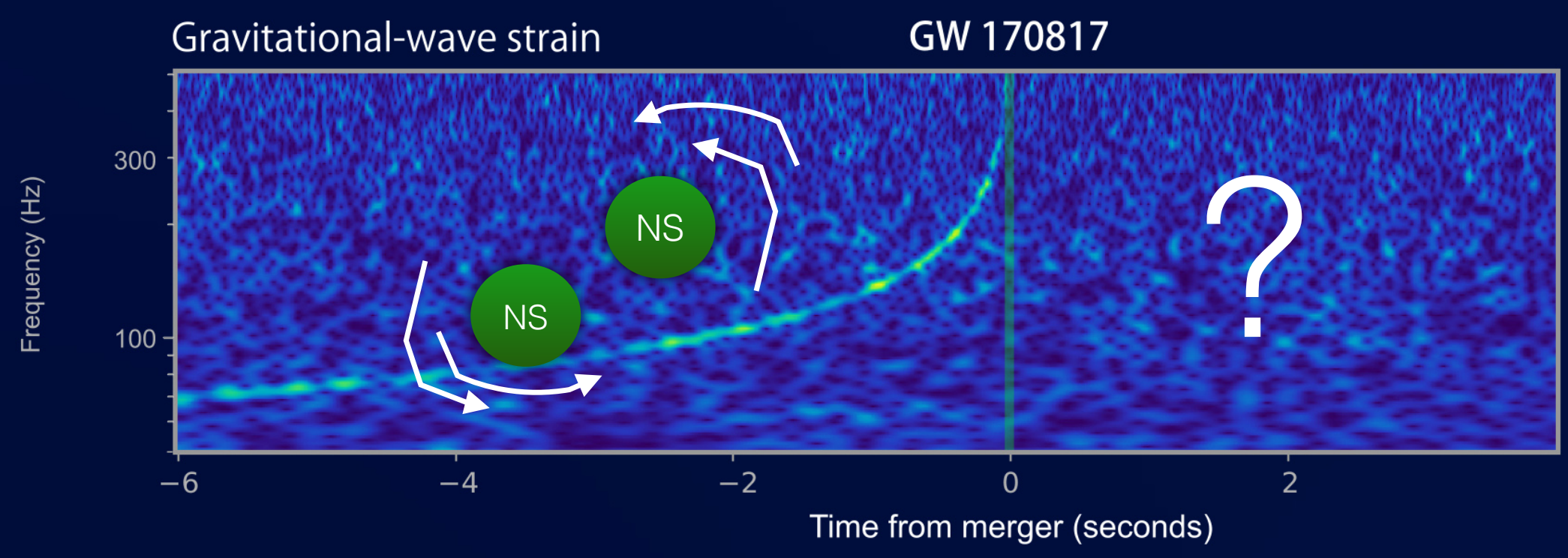

(c)2019 van Putten 


\section{GW170817: Extended Emission}

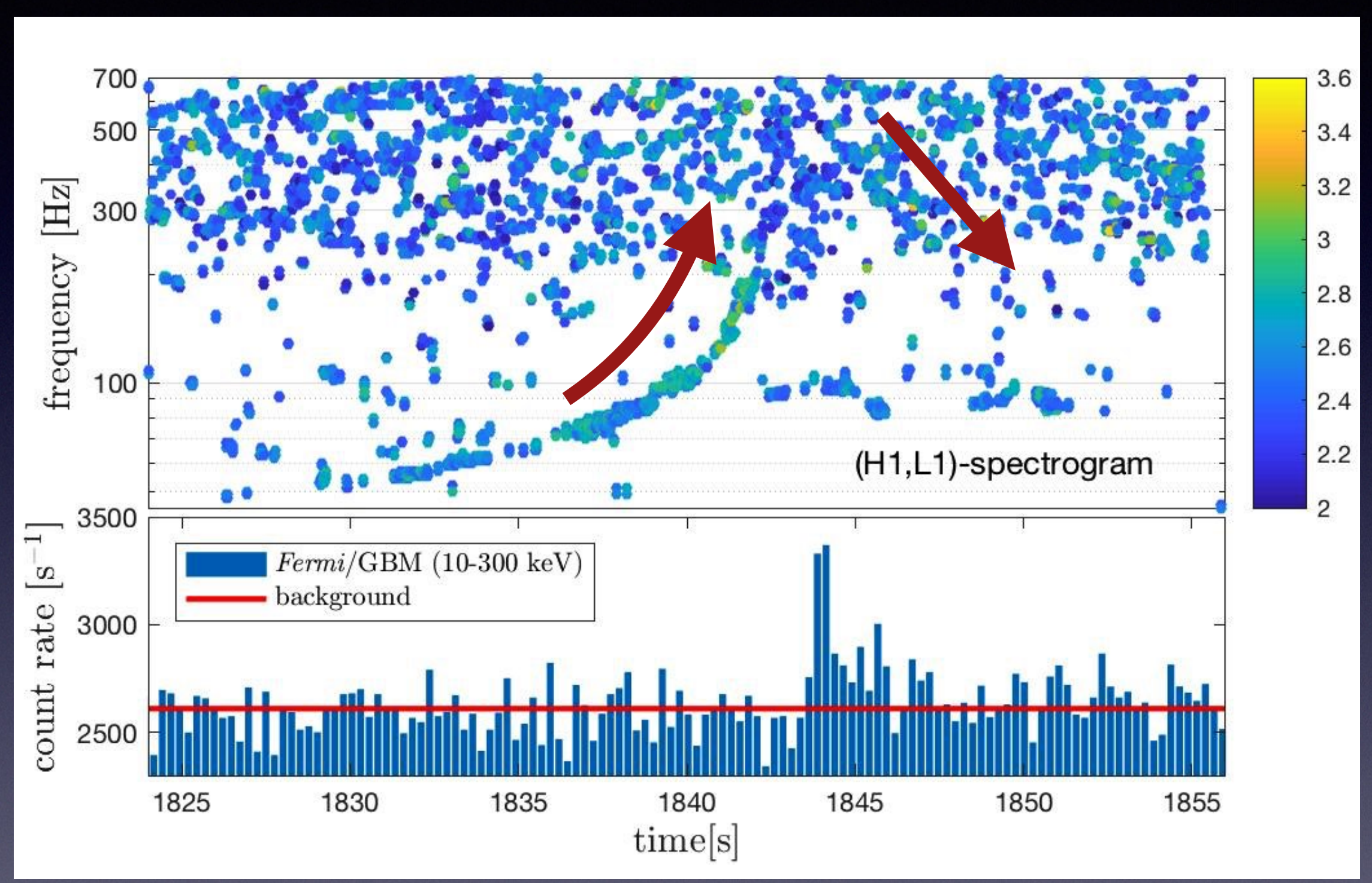

van Putten \& Della Valle, 2018, MNRAS Letters, 482, L46

JGW-G1808513-v1 https://gwdoc.icrr.u-tokyo.ac.jp/ 
Observational significance $4.2 \sigma(1: 40.000)$ by timing and amplitude (statistically independent attributes)

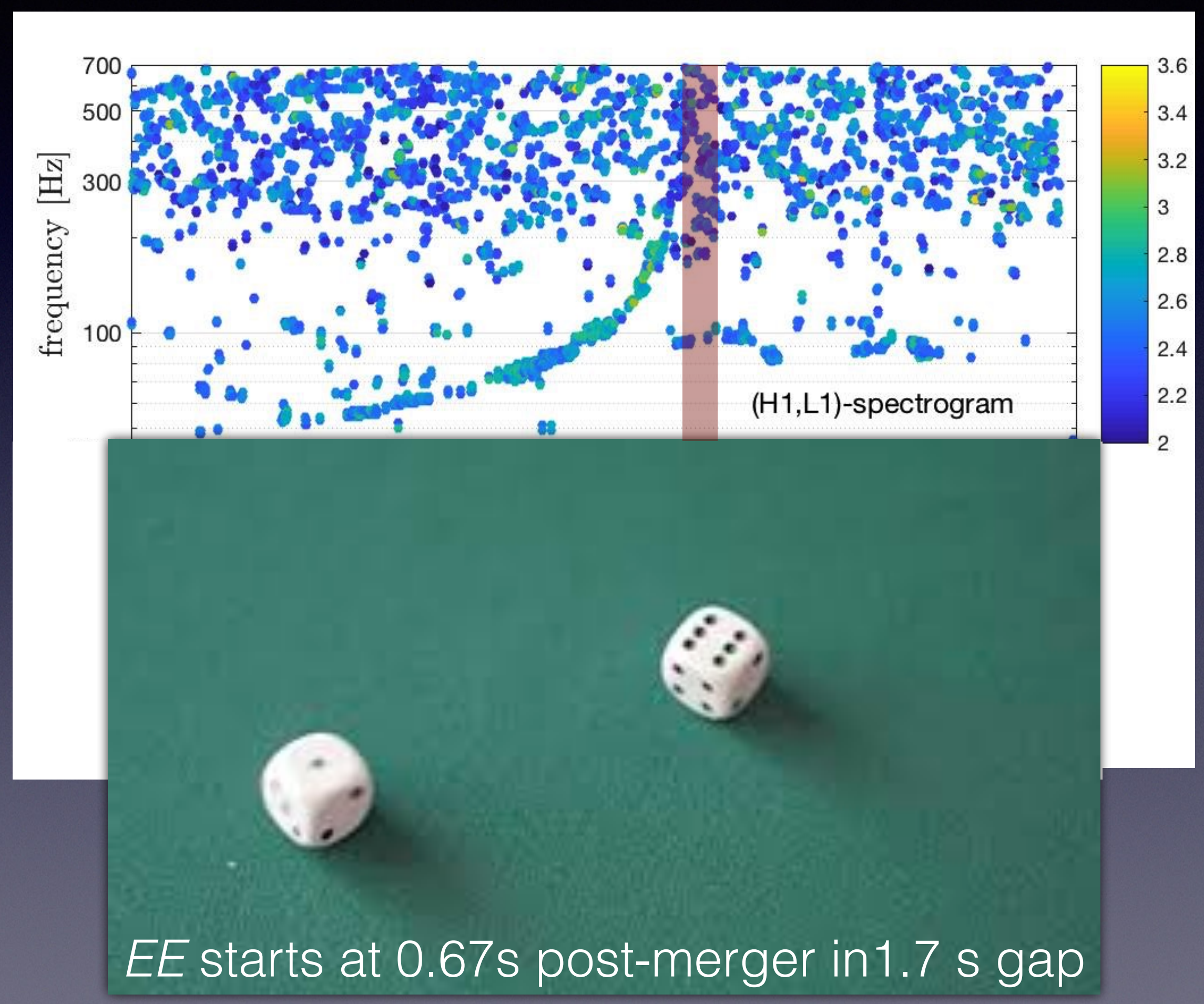


$E E$ - a descending chirp - radiates J HNS out to infinity.

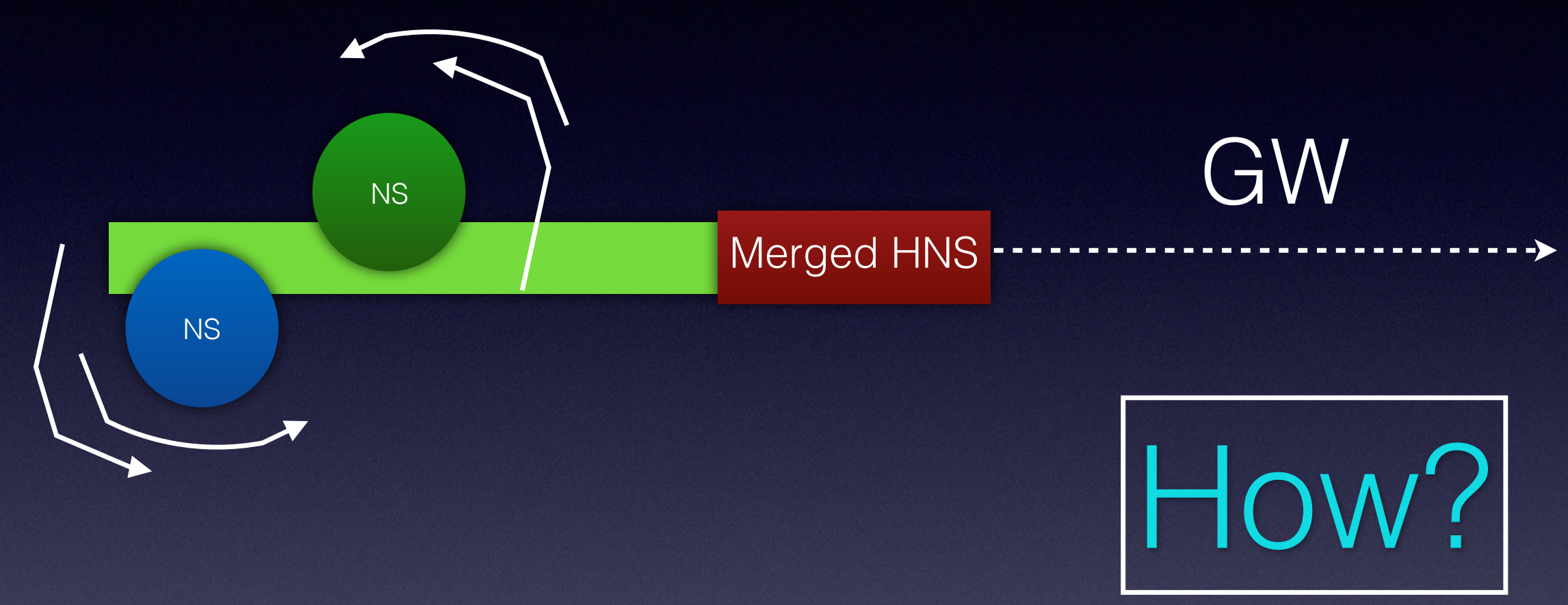

\section{YAHOO!

\section{Epic Crash of Neutron Stars Creates} 'Hypermassive Magnetar' 


\section{Calorimetry on Extended Emission}




\section{Calorimetry by Injection Experiment}

$$
\mathrm{GW} 170817 \quad \mathscr{E} \simeq ? \% M_{\odot} c^{2}
$$

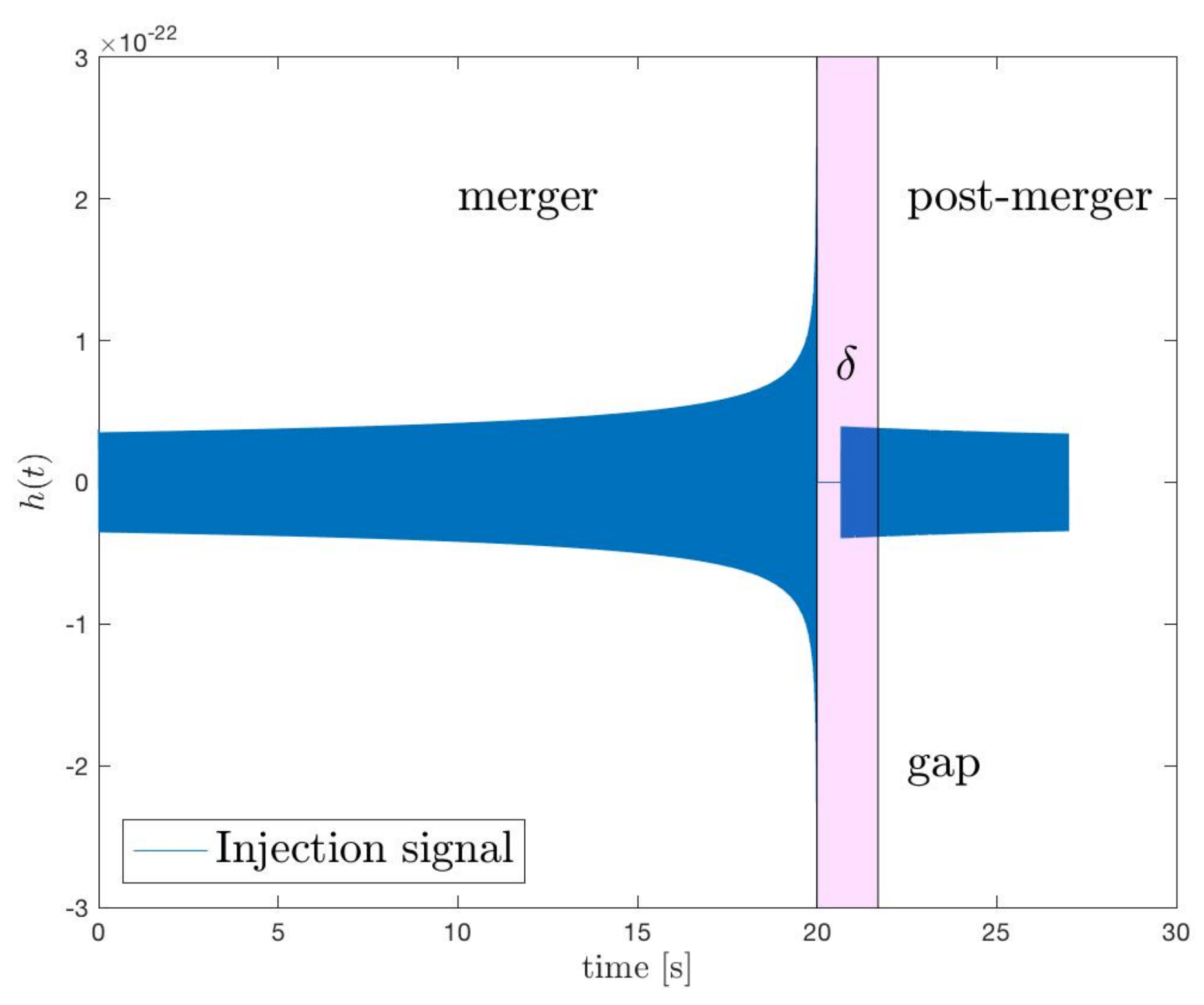


(H1,L1)-spectrogram merged by frequency coincidences

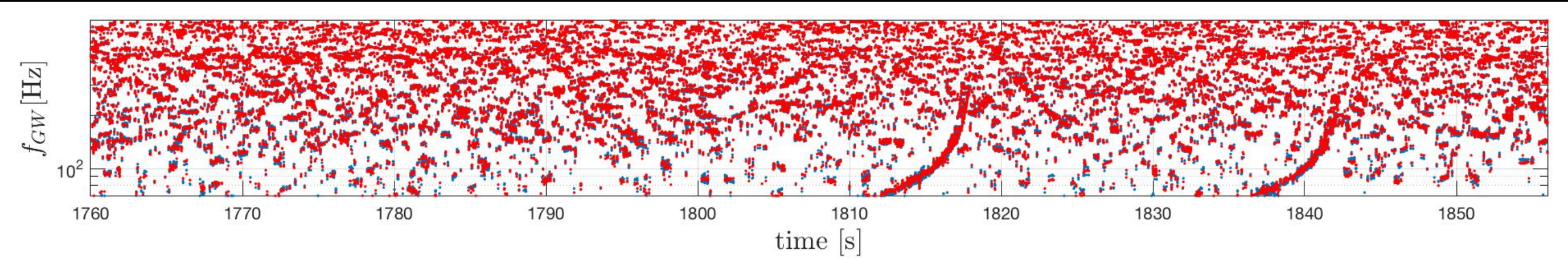

Injection GW170817EE

Matching ascending Granches: true-to-observed strain $\sim 0.7$

Matching descending Granches... 


\section{X-image analysis: matching peaks}

Scan over exponential features
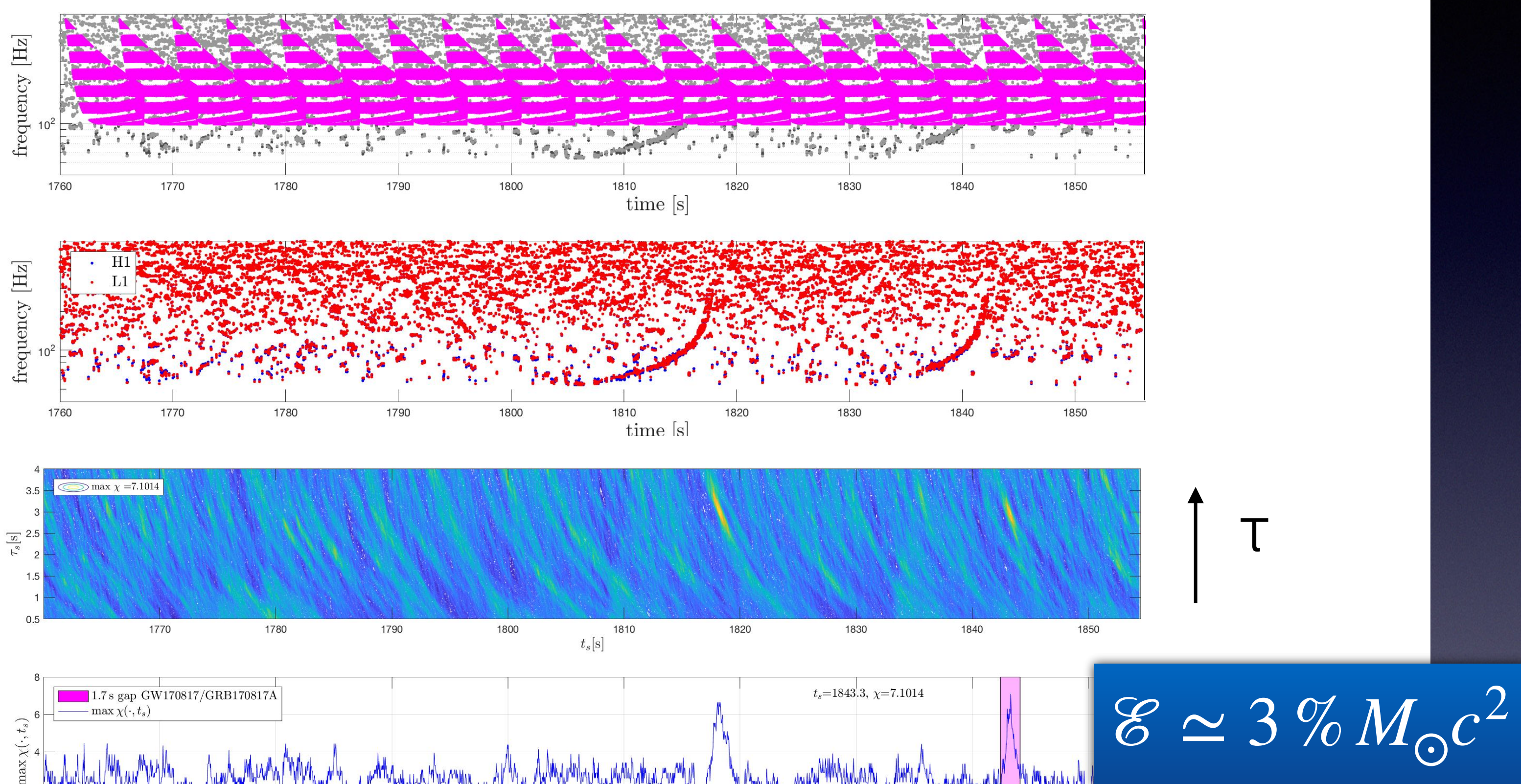

the

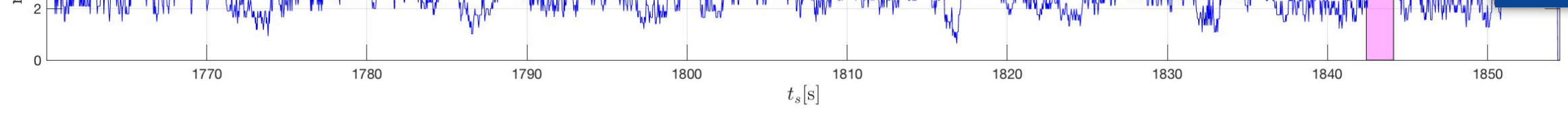

$$
t_{s} \simeq 0.67 \mathrm{~s}
$$




\section{Core-collapse greatly enhances $E_{J}$}

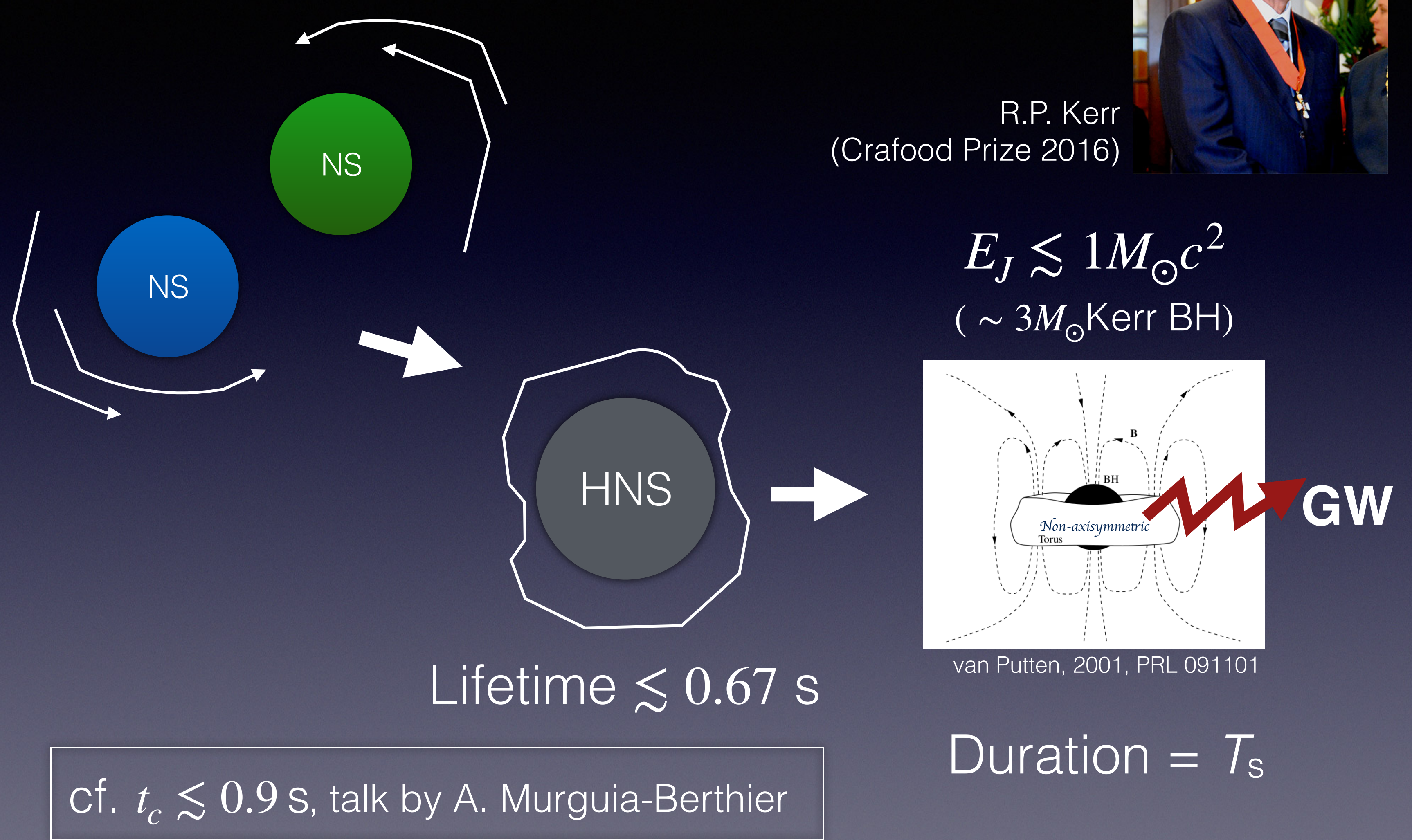




\section{Numerical solution equations of suspended accretion}

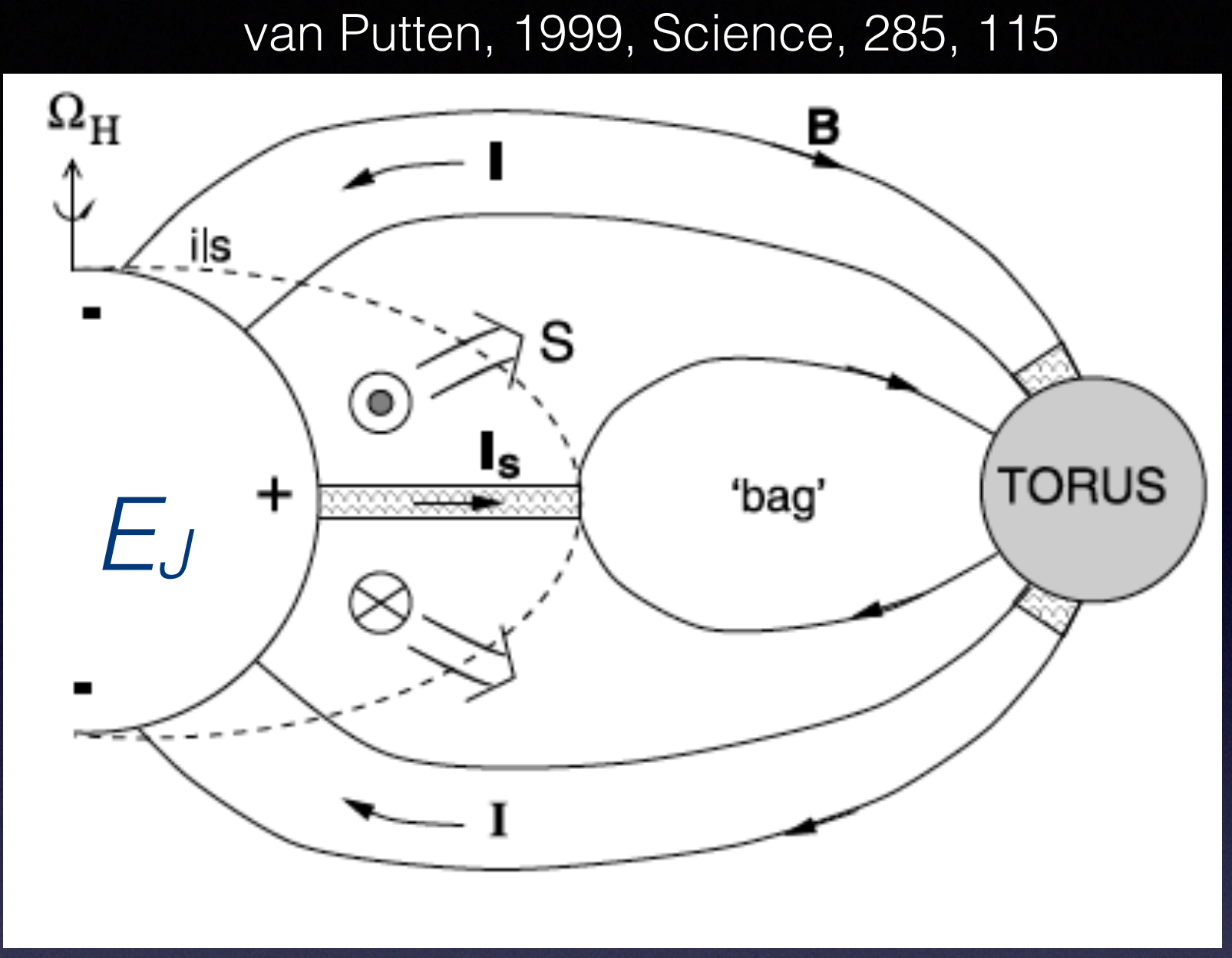

van Putten, Della Valle \& Levinson, 2019 (under review)

$$
\frac{L_{H}=-\dot{M}, T=-J_{H}}{K r_{I S C O}}
$$
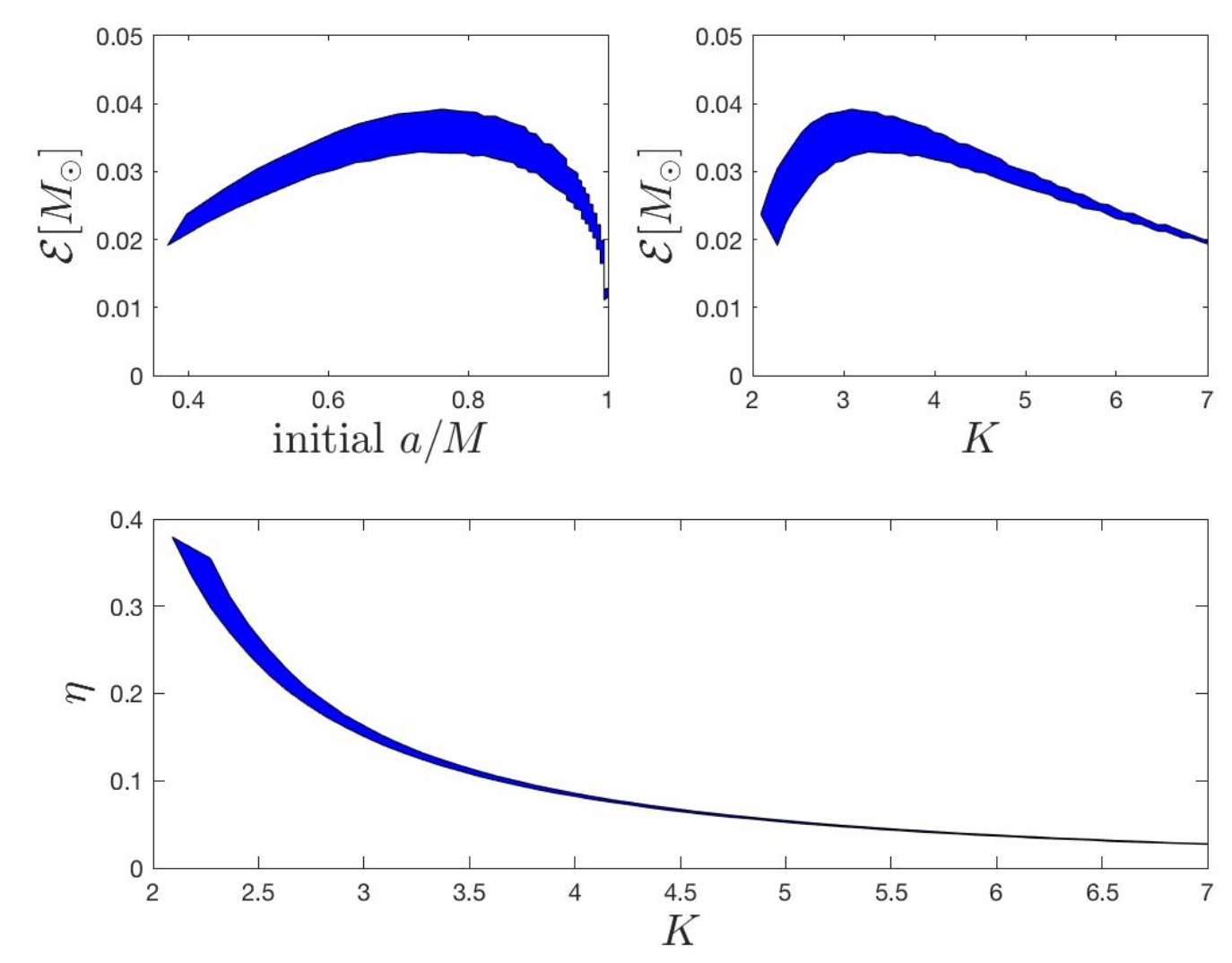

$$
\begin{gathered}
f_{G W, i}=650 \mathrm{~Hz} \text { (observed) } \\
K \simeq 3, \eta \simeq 15 \%
\end{gathered}
$$

$$
\mathscr{E} \simeq 3 \% M_{\odot} c^{2}
$$

by non-axisymmetric torus of $\sim 3 R i s c o$ about a low mass non-extremal $\mathrm{BH}(\mathrm{a} / \mathrm{M} 0.75)$

Theory and injection results agree. 
GW170817EE: complex sequence in three Acts

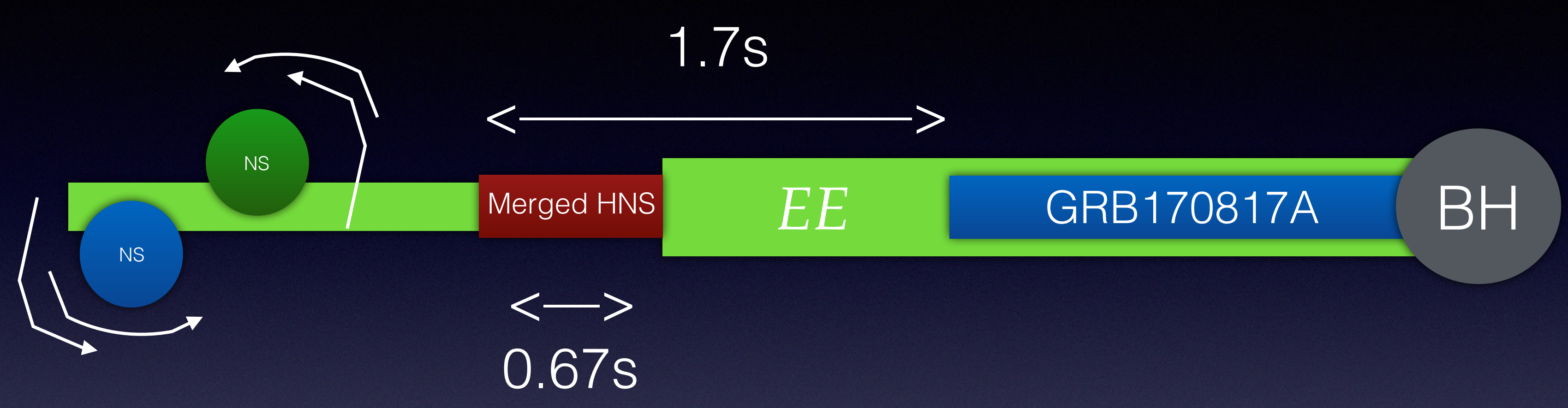

$$
\begin{aligned}
& E_{J}<1 \% M_{\odot} c^{2} \rightarrow E_{J} \simeq 0.3 M_{\odot} c^{2} \rightarrow \mathscr{E} \simeq 3 \% M_{\odot} c^{2} \\
& J=J_{H N S} \text { conserved in CC }
\end{aligned}
$$




\section{Short burst of MMEE}

Duration set by lifetime of spin:

$$
\begin{aligned}
& T_{s} \simeq 1.5 \mathrm{~s}\left(\frac{\sigma}{0.1}\right)^{-1}\left(\frac{z}{6}\right)^{4}\left(\frac{M}{M_{\odot}}\right) \\
& z=\frac{r}{M}, \quad \sigma=\frac{M_{T}}{M}
\end{aligned}
$$

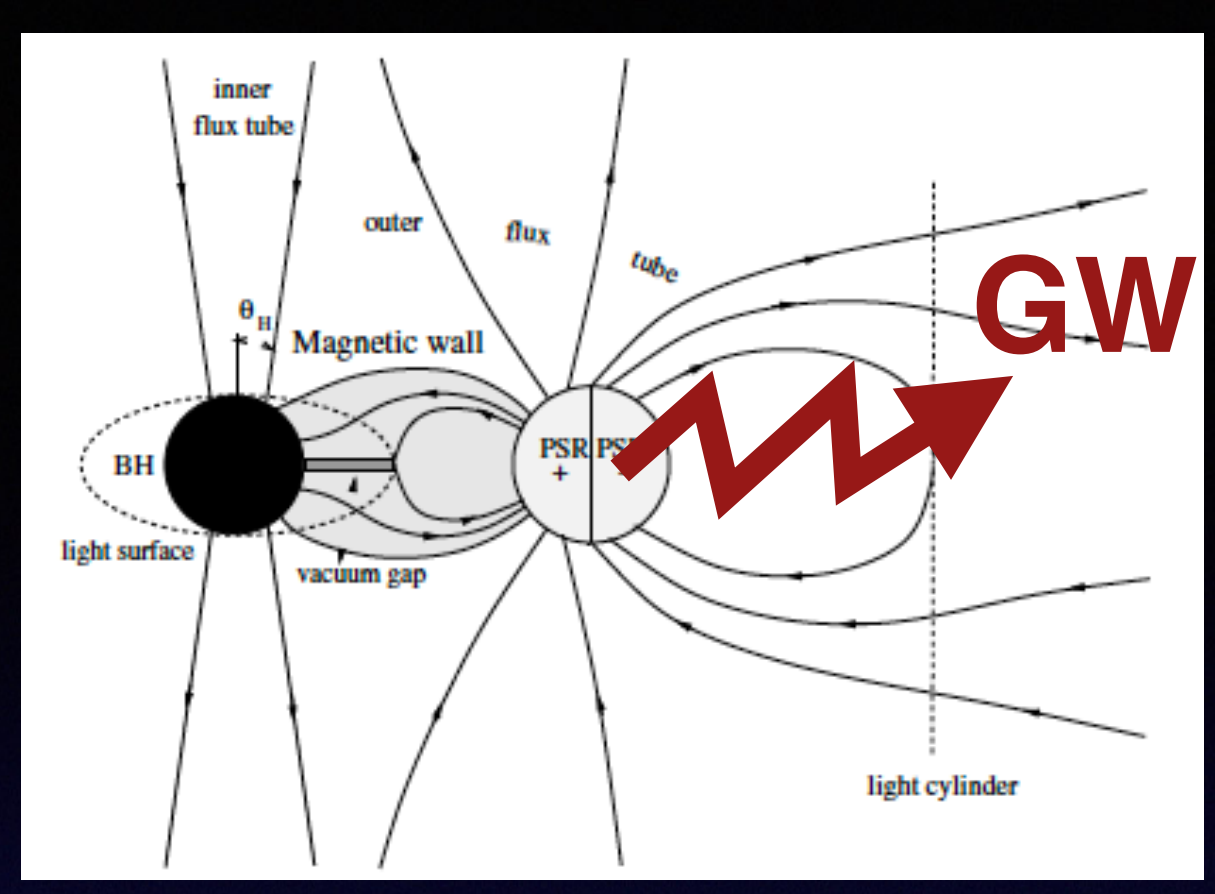

van Putten \& Levinson, 2003, ApJ, 584, 937

GRB170817A - kilonova:

$$
E_{j} \simeq \frac{1}{4 z^{4}} E_{J} \simeq 10^{50} \mathrm{erg} \quad E_{w} \simeq \eta^{2} E_{J} \simeq 10^{52} \mathrm{erg}
$$

Agrees with observational constrains (Mooley et al. 2019):

$$
E_{j} \simeq 10^{49-50} \mathrm{erg} \quad E_{k} \simeq 4.5 \times 10^{51} \mathrm{erg}
$$




\section{Multi-messenger Extended Emission}

GW170817EE: $\quad \mathscr{E} \simeq 3 \% M_{\odot} c^{2}$

Kilonova: $\quad 0.2 \% M_{\odot} c^{2}$

GRB170817A: $\quad 0.01 \% M_{\odot} c^{2}$ 